\title{
Neural signatures of recognition memory in 10- to 12-month-old infants
}

\author{
Szilvia Linnert ${ }^{\mathrm{a}, *}$, Brigitta Tóth ${ }^{\mathrm{b}}$, Márton Nagy ${ }^{\mathrm{c}, \mathrm{d}}$, Eugenio Parise ${ }^{\mathrm{a}}$, Ildikó Király ${ }^{\mathrm{c}}$ \\ a Department of Psychology, Lancaster University, Lancaster, United Kingdom \\ ${ }^{\mathrm{b}}$ Institute of Cognitive Neuroscience and Psychology, Research Centre for Natural Sciences, Hungarian Academy of Sciences, Budapest, Hungary \\ c Department of Cognitive Psychology, Eötvös Loránd University, Budapest, Hungary \\ d Doctoral School of Psychology, Eötvös Loránd University, Budapest, Hungary
}

\section{A R T I C L E I N F O}

\section{Keywords:}

Infant

EEG

ERP

Nc component

Familiarity

Frequency

Declarative memory

Recognition memory

\begin{abstract}
A B S T R A C T
Understanding memory mechanisms is crucial in the study of infant social and cognitive development. Here, we show that the Nc ERP component, known to reflect frequency-related attentional and/or memory processes, is a good candidate to investigate infant recognition memory. Previous paradigms have only investigated the effect of frequency during on-line stimulus presentation, but not during stimulus encoding. In this paper, we present a novel method for measuring the neural correlates of recognition memory and the 'degree' of familiarity in 10- to 12-month-old infants. During a familiarization phase, two images were presented frequently, while another two images were presented infrequently to the infants. In the test phase, the infrequent familiar, the frequent familiar, and the novel stimuli, were all presented with equal probability. We found larger Nc amplitudes following the familiar stimuli compared to the novel ones. The 'degree' of familiarity, on the other hand, did not modulate the Nc amplitude. These results can only be explained with memory-related processes, since in our paradigm the on-line presentation frequency did not vary. Furthermore, the lack of familiarization frequency effect suggests that the Nc might be a neural correlate of declarative memory.
\end{abstract}

\section{1. . Introduction}

The declarative memory, the capacity to recall and recognize facts and events, is fundamental to human cognition. It emerges by the end of the first year of life (Bauer et al., 2000), and is crucial in social and cognitive development. Learning culturally relevant information and establishing social relationship relies on declarative memory. For instance, it is possible to hypothesize that the capacity to strengthen social bonds or the ability to develop a certain attachment, is directly affected by the capacity to store information about that specific social partner (Bushnell, 2001). Learning how to use new artifacts relies largely on teaching episodes that need to be stored in memory (Casler and Kelemen, 2005; Tomasello, 1999) and often lasts a lifetime (cf. Gergely and Csibra, 2006). Therefore, memory plays a direct and crucial role in social and cognitive development. Research on memory development has provided evidence on long-term recall in children well before speech onset, demonstrating that this capacity shows marked variability in the first year despite its relevance for infants' social and cognitive development (Carver and Bauer, 1999).

There is some evidence that very young infants are capable of forming and recollecting complex memories that include what-wherewhen components of the original events (Richmond and Nelson, 2009; but see also Koski et al., 2013). However, investigating declarative memory represents a challenge in developmental research. An effective way would be to combine behavioral and neuroscience methods, such as the electroencephalogram (EEG) (Bauer et al., 2003; Carver et al., 2000). Nevertheless, this requires the identification of a memory-related EEG signature, such as an event-related potential (ERP) component.

Carver et al. (2000) and Bauer et al. (2003) found differences in the ERP responses to pictures of previously experienced "old" sequences and never-before-experienced "new" sequences in 9 month-old infants. Bauer et al. (2003) analyzed the Negative central (Nc) ERP component - a negative component over frontal and central scalp areas with a maximum peak between 300 and $700 \mathrm{~ms}$ after stimulus onset (Courchesne et al., 1981). Novel stimuli elicited greater Nc amplitude compared to familiar images, indicating that the infants encoded the events. This result suggests that the Nc ERP component is a reliable indicator of some sort of recognition or familiarity in infants. Furthermore, the Nc component has been shown to be sensitive to socially relevant stimuli, such as the mother's face (de Haan and Nelson, 1997, 1999) and the infants' favorite toy (de Haan and Nelson, 1999).

Though the Nc amplitude has been previously suggested to be sensitive to memory processes, the on-line stimulus presentation

\footnotetext{
* Corresponding author:Correspondence to: Department of Psychology, Fylde Collage, Lancaster University, Lancaster LA1 4YF, United Kingdom.

E-mail address: s.linnert@lancaster.ac.uk (S. Linnert).
} 
frequency also seems to modulate it. Courchesne et al. (1981) presented images of female faces with different frequency rates (88\% standard vs. $12 \%$ oddball trials). The Nc component was significantly larger for the oddball compared to the standard face. The authors have suggested two interpretations. First, the Nc might reflect differences in stimulus presentation frequency: the larger Nc amplitude reflects the detection of rare stimuli. Such on-line frequency change monitoring indicates a bottom-up, attentional process. Second, the Nc amplitude might reflect the strength of the memory trace. The frequently presented stimulus became more and more familiar during the experiment. Therefore, a smaller Nc amplitude reflects stronger memory trace for the frequent event, and a higher Nc amplitude reflects weaker memory trace for the rare event. This second explanation suggests a top-down, memory-related process. Nevertheless, according to both explanations the Nc seems to be a neural correlate of some sort of frequency based encoding process (Ackles, 2008).

The following studies manipulated both the familiarity and on-line presentation frequency of the stimuli (e.g. Nelson and Collins, 1992, 1991; Reynolds et al., 2010; Reynolds and Richards, 2005; Richards, 2003). In all these studies, infants were familiarized with some of the stimuli, but not with all of them. During the oddball procedure, the familiarized stimuli were shown in either $60 \%$ or $20 \%$ of the trials, while novel stimuli were presented in the remaining $20 \%$ of the trials. As a result, infants were exposed to frequent familiar, infrequent familiar, and infrequent (trial-unique) novel stimuli. Reynolds and Richards (2005) found larger Nc amplitude following the infrequent novel stimuli compared to frequent familiar images in 4.5-, 6- and 7.5month-old infants. However, the Nc amplitude for the infrequent familiar stimuli did not differ from the Nc amplitude for either the frequent familiar or the novel images. Such differences might signal either memory-related processes (familiar vs. novel difference) or the effect of presentation frequency (frequent vs. infrequent difference). On the other hand, the results also indicated that the on-line presentation frequency alone does not modulate the Nc amplitude. A control group of infants was familiarized with images that were not used later in the oddball procedure. Therefore, all stimuli were new for this group as they were presented with frequent novel, infrequent novel, and infrequent trial-unique stimuli. The Nc amplitude did not differ between stimulus types, indicating that the Nc amplitude cannot simply reflect bottom-up, presentation-frequency dependent, attention or orientation effects (cf. also Ackles, 2008). Therefore, top-down factors, such as detection of something being familiar, might modulate the Nc amplitude.

In all previous experiments, the effect of frequency on the Nc has been investigated only by manipulating the on-line stimulus presentation probability. But according to the memory-based explanation of the Nc (Courchesne et al., 1981), the Nc amplitude might rather be sensitive to a frequency-based stimulus encoding process (Ackles, 2008). Memories with a what-where-when component are encoded based on a unique, single event, whereas familiarity-based recognition doesn't necessarily rely on a single exposure. Previous studies on the Nc have not investigated the differential contribution of stimulus frequency to memory processes. We propose to systematically vary both the frequency and the familiarity components of the stimuli during the encoding (familiarization phase), while keeping constant the on-line stimuli presentation frequency (test phase). Such a manipulation allows us to study the effect of previously encountered frequency differences - in other words, the 'degree' of familiarity - on the amplitude of the Nc component, with no confound of on-line presentation frequency differences.

Our new ERP paradigm is articulated into a familiarization and a test phase. In the familiarization phase, the infants saw novel images of colorful toys. Half of the stimuli appeared frequently (nine times each), the other half infrequently (three times each). In the test phase, while we recorded the EEG, infants saw three kinds of stimuli, all presented with equal probability: the two kinds of familiar stimuli (infrequent familiar and frequent familiar) as well as novel (non-familiar) stimuli. Both familiarity (familiar vs. novel stimuli) and frequency (frequent familiar vs. infrequent familiar stimuli) effects can only be ascribed to the familiarization phase, revealing the role of memory encoding. As a result, any modulation of the Nc component can only depend on the 'degree' of stimuli familiarity (memory-related top-down processes) and cannot reflect a basic change due to frequency monitoring (bottom-up attentional processes). We expect Nc amplitude differences between the frequent familiar and novel stimuli. However, the question is whether the Nc amplitudes for the infrequent familiar stimuli would be more similar to the frequent familiar or novel images.

\section{Methods}

\subsection{Participants}

The experiment took place in the EEG Lab at the Department of Cognitive Psychology, Eötvös Loránd University, Budapest, Hungary. All parents were informed about the experiment before they volunteered and signed a consent form. The Ethical Committee of the Institute of Psychology at the ELTE University approved the research project and the experimental procedure. Fifteen infants (6 females), full-term and normally developing, were included in the final sample. The mean age was 11 months 15 days ( $S D=24.56$ days). Eleven additional infants participated, but were excluded from the final sample, because they did not provide enough artifact free segments to compute the ERPs $(\mathrm{N}=6)$; because of equipment failure $(\mathrm{N}=3)$ or fussiness $(\mathrm{N}$ $=2$ ). Infants were together with their parent during the experiment and they received a toy for volunteering.

\subsection{Stimuli and materials}

The stimuli were eight colored pictures of novel toys (see Fig. 1). In order to ensure that all stimuli were equal in saliency, we ran a separate eye-tracker pre-test study on a broader set of stimuli (30 toy pictures). Thirty-seven infants were involved in the pre-test (mean age $=12$ months 10 days, $S D=136.45$ days, age range: 9 months -2 years). We used Clearview 2.5.1 software to display random image pairs for $2700 \mathrm{~ms}$ and a Tobii $\times 50$ eye-tracker (Tobii Technology, Sweden) to record eye-movement data. We calculated the average fixation time (the quotient of total looking time and number of fixations on the toy) for each object and there were no significant differences from the overall average fixation for the toys used in the EEG experiment (onesample $t$-tests, test value $=69.9 \mathrm{~ms}, p<.05$ ). This indicates that none of the toys presented in the EEG experiment was more interesting for the infants, ruling out saliency related effects.

The stimuli in the EEG experiment were colored pictures of 8 toys with a hand holding them against a black background (height: 400 pixels, width: 300 pixels). We included the hand to suggest a social context and increase infants' attention. During the experiment the stimuli appeared on a black background in the center of a 17" LCD computer screen with a $60 \mathrm{~Hz}$ refresh rate and $1280 \times 1024$ pixels resolution at a viewing distance of approximately $70 \mathrm{~cm}$. We used Eprime 2.0 software (Psychology Software Tools, Pittsburgh, PA) for stimuli presentation. We monitored the infant's gaze on-line with a video camera placed near the monitor. When children looked away from the screen we presented an attention grabber (a short animation of a black and white chessboard pattern shown in the middle of the screen, coupled with an animal sound) in order to re-orient them back to the monitor. Infants were seated in the parent's lap during the whole experiment.

\subsection{Procedure}

Fig. 2 shows the schematic diagram of the experimental design. The experiment consisted of two phases: a familiarization and a test (EEG) 

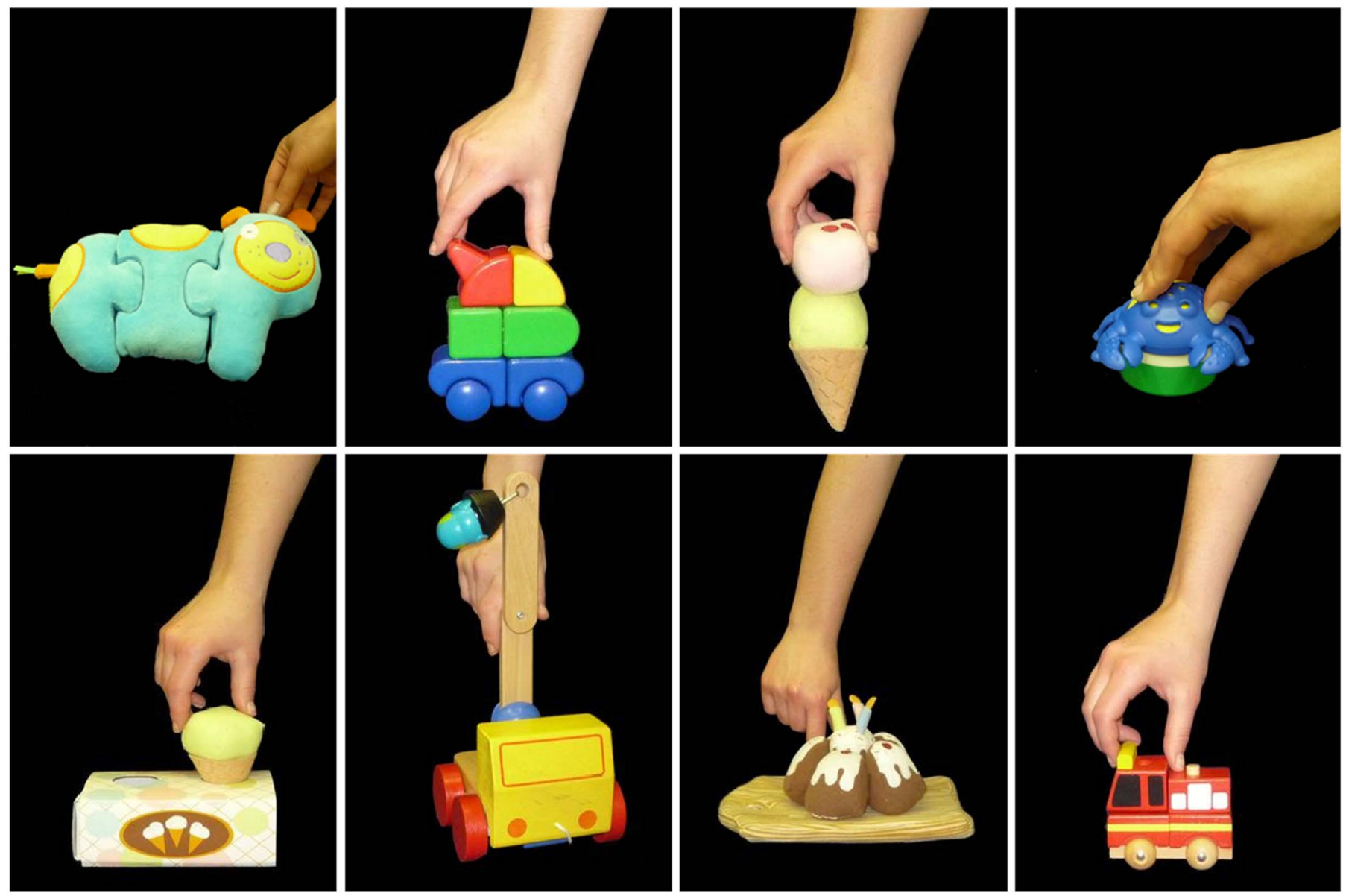

Fig. 1. Stimuli. The two frequent, two infrequent and two novel images were randomly chosen for each participant.

phase. Each infant saw six images, randomly chosen out of the set of eight. In the familiarization phase, we familiarized the participants with four images, and in the test phase, they saw two additional, randomly selected pictures as novel stimuli.

In the familiarization phase, we repeatedly presented the four images with different probabilities: we showed two images for $75 \%$ of the trials and the other two images for the remaining $25 \%$ of the trials. The familiarization phase consisted of 24 trials, where each frequent toy was presented nine times and each infrequent toy was presented three times $(2 \times 9+2 \times 3=24)$. A single trial of the familiarization phase began with a white fixation cross displayed in the center of the black screen for a random period between 300 and $600 \mathrm{~ms}$ followed by a stimulus presentation for $6000 \mathrm{~ms}$. The familiarization phase lasted approximately $3 \mathrm{~min}$. The presentation order was pseudorandom with the following constraints: we delivered the trials in three blocks, in each block we presented the infrequent images once, and the frequent images three times. Within a block, the order of the presented images was randomized. During a 5-10 min long break between the experimental phases, the infants could play.

In the test phase, we presented all four previously shown images together with two novel stimuli. We displayed all frequent familiar, infrequent familiar and novel stimuli with equal probability (33.3\%). We presented the stimuli in blocks of six trials where all images appeared once. The presentation order was random within a block. A single trial started with a white fixation cross displayed in the middle of the black screen for a random period between 300 and $600 \mathrm{~ms}$ followed by a stimulus presentation for $1500 \mathrm{~ms}$. The screen was blank for $600 \mathrm{~ms}$ following the stimulus presentation. The test phase ended when the child became fussy or their attention could no longer be directed to the screen. During this phase, we recorded both EEG and video (synchronized with the EEG).

\subsection{EEG recording and analysis}

To record the EEG, we used an EGI (Electrical Geodesics Incorporated, Eugene, OR) GES300 EEG system with NetAmps300 amplifier and 128 channel HydroCel Geodesic Sensor Nets (HCGSN). The EEG was recorded with NetStation software (Electrical Geodesics Incorporated, Eugene, OR). We kept the impedances below $75 \mathrm{kOhm}$. During the recording, the EEG was referenced to vertex $(\mathrm{Cz})$. The sampling rate was $1000 \mathrm{~Hz}$, with an antialiasing low-pass filter of $400 \mathrm{~Hz}$. We recorded vertical eye movements from the electrodes above the eyes (channels 8 and 25 on HCGSN net).

The analysis of EEG data was performed using NetStation software. The continuous EEG signal was band-pass filtered between .3 and100 Hz. The EEG was segmented into epochs of $1600 \mathrm{~ms}$ long (separately for each stimulus: frequent familiar, infrequent familiar and novel), starting from $100 \mathrm{~ms}$ before the onset of the stimulus, to $1500 \mathrm{~ms}$ after stimulus onset. Off-line, we marked and excluded from further analysis all those trials where the infant was not attending to the screen. We automatically rejected eye-blinks and bad channels if the average amplitude in an $80 \mathrm{~ms}$ moving window was exceeding $\pm 55 \mu \mathrm{V}$ at EOG channels or $\pm 200 \mu \mathrm{V}$ at any other channel. In addition, each individual epoch was visually inspected and we rejected further epochs or channels. If an epoch contained less than 13 bad channels, we interpolated the signal of the bad channels using the remaining channels for spherical spline interpolation. We rejected those epochs where more than 13 channels contained artifacts. We excluded all infants with less than eight artifact free segments per condition. For each infant and for each condition (frequent familiar, infrequent familiar and novel) we 


\section{Familiarization \\ phase}

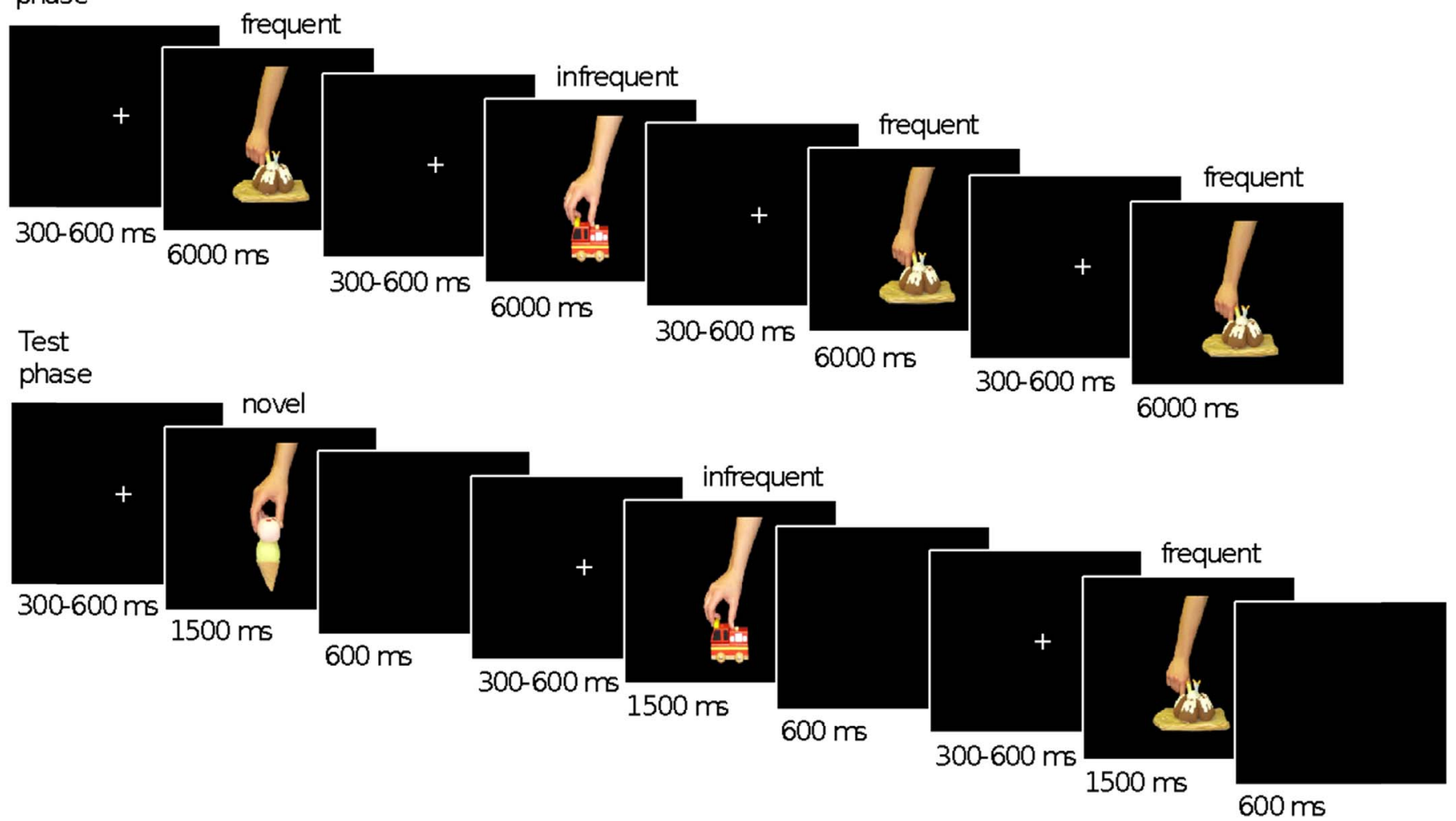

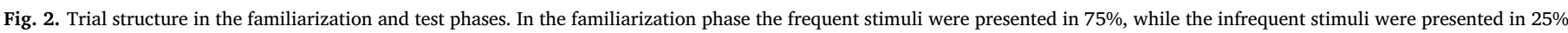
of the trials. In the test phase all three stimulus types were shown with equal probability.

computed average ERPs on artifact free segments. The number of average artifact free segments per infant didn't differ between the three conditions (frequent familiar: $M=17.27, S D=6.90$, range: 8-29; infrequent familiar: $M=17.20 ; S D=6.61$, range: $10-30$; novel: $M=$ $17.25, S D=7.03$, range: $8-32$; ANOVA $F(2,28)=.006 ; p=.994$; $\eta 2<.001$ ). We used the average voltage in the $100 \mathrm{~ms}$ pre-stimulus period to baseline correct the ERPs and re-referenced them to average reference. We used EEGLAB (v. 9..5.6b) toolbox with $30 \mathrm{~Hz}$ low-pass display filter to display grand average ERPs.

We defined the Nc component (based on the grand average waveforms) as the most prominent negative component peaking between the 300 and 700 ms post-stimulus interval. Based on Reynolds and Richards (2005) we defined a frontal midline ROI, including the Fz and the surrounding electrodes $(4,10,16,18,19)$ and a central midline ROI, including $\mathrm{Cz}$ and the surrounding electrodes $(7,31,55,80,106)$. We computed the mean amplitudes between 360 and 560 ms post-stimulus intervals for the two ROIs and the three stimulus conditions separately. We selected the time window based on visual inspection of the grand average waveforms (see Fig. 3). We used SPSS software (version 22) for statistical analysis of the Nc amplitude, performing a $3 \times 2$ ANOVA with Grand averages (frequent familiar, infrequent familiar and novel) and Area (frontal and central ROIs) as within subjects' factors on the mean Nc amplitudes. When necessary, we adjusted the $p$-values using the Greenhouse-Geisser correction for violations of the assumption of sphericity.

\section{Results}

Fig. 3 shows the grand average ERP waveforms and its scalp distribution for the three types of stimuli. Fig. 4 shows the mean amplitudes and standard errors. We found a significant main effect of Stimulus type $(F(2,28)=3.84 ; p=.034, \eta 2=.215)$. The Nc amplitudes were larger for frequent familiar $(M=-4.43 \mu \mathrm{V}, S D=3.63 \mu \mathrm{V})$ and infrequent familiar stimuli $(M=-3.91 \mu \mathrm{V}, \mathrm{SD}=5.28 \mu \mathrm{V})$, compared to novel stimuli $(M=-1.80 \mu \mathrm{V}, \mathrm{SD}=4.36 \mu \mathrm{V})$. The post-hoc $t$-tests revealed that the Nc response evoked by novel stimuli was smaller compared to both frequent familiar $(t(14)=2.58, p=.022)$ and infrequent familiar $(t(14)=2.254, p=.041)$ stimuli. The difference between frequent and infrequent familiar stimuli was not significant $(t$ $(14)=.52, p=.629)$. There was no significant main effect of Area $(F$ $(1,14)=.115 ; p=.739, \eta 2=.008)$ or interaction of Area by Stimulus type $(F(2,28)=.637 ; p=.536, \eta 2=.044)$. Additionally, we ran nonparametric tests for the main results we found. Twelve out of 15 infants showed a more negative Nc for frequent familiar vs. novel stimuli (Wilcoxon's $Z$ test: $Z=2.215, p=.027$ ); 11 out of 15 infants showed larger Nc for infrequent familiar vs. novel stimuli (Wilcoxon's $Z$ test: $Z$ $=1.988, p=.047$ ).

The Nc amplitude difference changes with age (Bauer et al., 2006). In order to test whether in our sample the Nc amplitude difference was modulated by age, we split the sample into two groups, according to the infants' age (younger group: $N=8$; older group: $N=7$ ). We ran an additional, $3 \times 2 \times 2$ ANOVA with Stimulus type (frequent familiar, infrequent familiar and novel) and Area (frontal and central ROIs) as within subjects factors and Age (young and old) as a between subjects factor. We found only a significant main effect of stimulus type $(F(2,26)$ $=4.282 ; p=.025, \eta 2=.248)$. There was no main effect of Age $(F(1$, $13)=2.968 ; p=.109, \eta 2=.186)$, neither Age by Stimulus type interaction $(F(2,28)=1.77 ; p=.189, \eta 2=.12)$.

\section{Discussion}

The present study aimed to investigate the differential contribution of the presentation frequency on memory processes. We manipulated the degree of familiarity in 10 to 12-month-old infants and measured 
a)

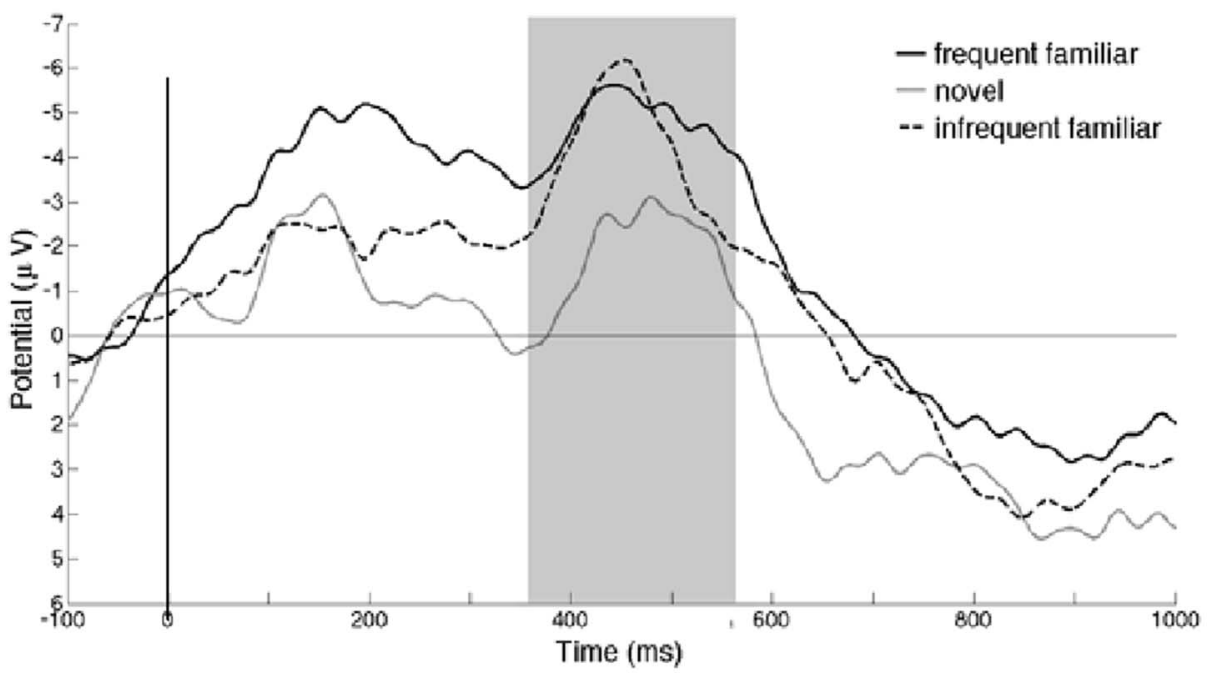

Fig. 3. a), Group-average ( $\mathrm{N}=15)$ ERPs elicited for frequent, infrequent and novel trials triggered from visual image (average waveform at frontal and central ROI). Grey box mark the measurement windows for the Nc. b), Average scalp distribution of the grand average ERPs between 360 and $560 \mathrm{~ms}$ for frequent, infrequent and novel trials.

b)
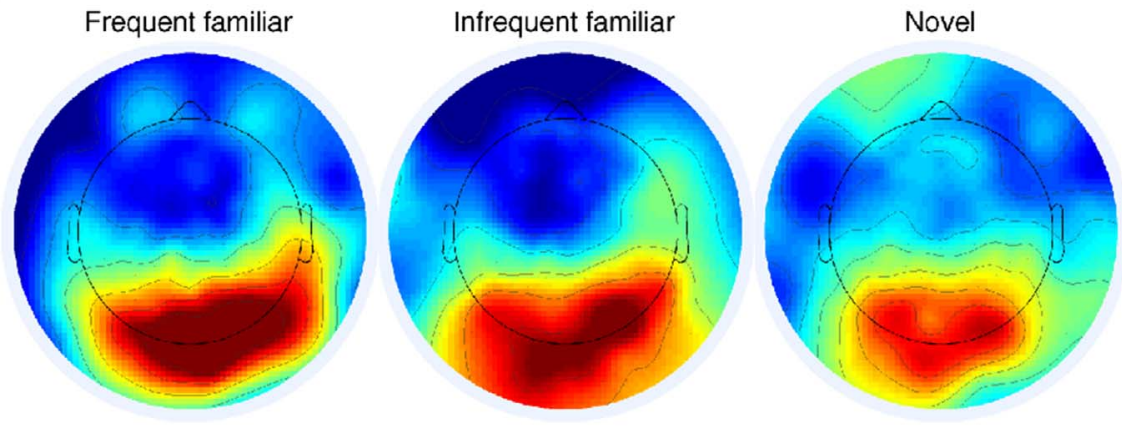

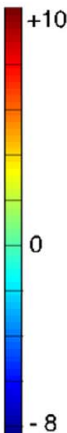

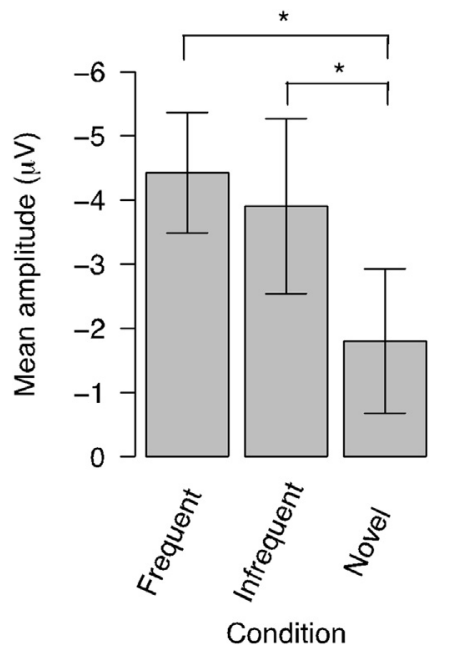

Fig. 4. Mean Nc amplitudes for frontal and central ROIs collapsed together. The columns represent the mean $\mathrm{Nc}$ amplitudes, the error bars represent SE. The Nc amplitude for the novel images differed both from the Nc amplitudes for the frequent and infrequent stimuli.

the Nc ERP component in a passive viewing recognition task. Unlike other studies, where the effect of on-line presentation frequency was tested, we manipulated the presentation frequency during the familiarization (memory encoding) phase. During the test phase, we compared event-related brain activity elicited by images with different degrees of familiarity (infrequent familiar, frequent familiar and novel), but with equal on-line presentation frequency. This allowed us to measure memory-related top-down differences, rather than low-level effects of on-line presentation frequency. The results revealed that the amplitude of the Nc component over the midline frontal and central electrodes was clearly sensitive to a familiarity - novelty distinction. The Nc amplitude was higher following familiar images compared to novel pictures. Yet, the presentation frequency difference that originated in familiarization did not modulate the amplitude of the $\mathrm{Nc}$ component.

Usually, a larger Nc amplitude is associated with novel or less frequent stimuli (Bauer et al., 2003; Courchesne et al., 1981; Reynolds and Richards, 2005), whereas we found larger Nc amplitudes for familiar relative to novel images. Nevertheless, some studies have also found more negative Nc amplitudes following familiar stimuli (de Haan and Nelson, 1997, 1999). Below, we will discuss three factors that can possibly modulate the differences in Nc amplitudes: (1) attention allocation processes; (2) effect of repeated presentation; (3) contextual effects of stimulus presentation.

Presuming that higher Nc amplitude reflects higher attention (Reynolds and Richards, 2005), a larger Nc amplitude following novel images is in line with the assumption that infants prefer novel stimuli (Fantz, 1964). However, infants have also been shown to prefer familiar stimuli in the beginning of the encoding process (e.g. Hunter et al., 1982). Therefore, in the early phase of stimulus processing, a familiar image might be more attention catching for infants. Roder et al. (2000) showed systematic familiar image preferences for objects and faces in the beginning of the encoding process, before the presence of a novelty preference. The authors explained this as a "phase of selective attention to the target (partially encoded familiar) stimulus" (Roder et al., 2000, pp. 502). Encoding processes could also change with age. Bauer et al. (2006) interpreted the change of the Nc effect between 9 and 10 months as differential allocation of processing resources between familiar and 
novel images. While 9-month-old infants devoted more attention to the novel stimuli, 10-month-olds devoted more attention to the familiar stimuli (Bauer et al., 2006). This explanation also indicates the role of attention during encoding. In our study, age didn't modulate the Nc differences between stimulus types. On the other hand, a process that allocates attention to partially encoded familiar stimuli could explain our results, as all the toys were novel to the infants at the beginning of the experiment. Nevertheless, it is important to emphasize that such attention-allocation requires top-down processes, because it is based on recognition.

The way frequency differences were implemented in previous studies could also be a factor modulating the direction of the Nc effect. Some studies presented the novel stimuli repeatedly (e.g. Bauer et al., 2006, 2003; de Haan and Nelson, 1997, 1999), while in other studies the novel stimuli differed on each trial (Nelson and Collins, 1992, 1991; Reynolds and Richards, 2005; Richards et al., 2010). A trial-unique stimulus appears only once in an experiment, therefore, the frequency of such item is very low. Wiebe et al. (2006) showed familiar-repeated, novel-repeated and trial-unique novel stimuli with equal probability to 9 month-olds and compared the Nc amplitudes from the early and late trials. Repeated presentation decreased the Nc amplitude from early to late trials, while the Nc did not change for the trial-unique novel stimuli. In this case, a larger Nc for trial-unique novel stimuli might simply be an effect of decreased Nc amplitude following the repeatedly presented stimuli. In our study this cannot be the case, because all stimulus types were presented repeatedly.

Finally, the context in which the stimuli are presented can also modulate the Nc amplitude. de Haan and Nelson (1997) presented the mother's face and a stranger's face to 6-month-old infants. The Nc amplitude was larger for the mother's face only if the stranger's face was dissimilar compared to the mother's (Experiment 1). If the two faces were similar to each other, the Nc amplitude was more negative for the stranger's (novel) face (Experiment 3). The authors suggested that the context in which the familiar face is seen might facilitate different neural processes (de Haan and Nelson, 1997). The role of context is also supported by other studies in infants (Ackles and Cook, 1998; Reynolds and Richards, 2005) and in adults (Richmond et al., 2007). Infants cannot be given any task instructions, therefore the question arises, whether they interpreted the procedure as a recognition task or not, and the context in which the stimuli appear might modulate this aspect. Repeated presentation of novel stimuli might facilitate the recognition of familiar objects, whereas trial-unique novel stimuli might capture more attention than the repeatedly presented familiar ones.

Overall, there is emerging consensus that a larger Nc amplitude reflects stronger allocation of attention. However, as we have shown above, several factors modulate the process of capturing attention. The familiarity effect in our study is necessarily based on the prior image presentation (familiarization phase) and cannot be the result of different presentation frequency during the test phase. Therefore, in this study the amplitude of Nc reflects recognition memory processes. A stronger attention orientation response to the familiar images seems to be rational.

We did not find a difference in terms of the Nc magnitude between frequent familiar and infrequent familiar stimuli. However, in our study even the infrequent stimuli were presented 3 times for $6 \mathrm{~s}$ ( $18 \mathrm{~s}$ overall) during the familiarization phase. Posing the question, is a single presentation sufficient for recognition? In infant memory research, it is a challenge to separate familiarity-based recognition (with no whatwhere-when components of the original event) from declarativememory based recognition (when the original event is actually recalled). Memories with a what-where-when component are encoded based on a unique, single event, whereas familiarity-based recognition does not necessarily rely on a single exposure. Our results indicate that the Nc component is not sensitive to presentation frequency during encoding - at least for the presentation frequencies we used. This suggests that the Nc does not merely signal the degree of familiarity and that the Nc component might also be a valid candidate to signal declarative memory processes. However, further studies should investigate this question using only a single exposure of an 'event'.

In conclusion, our results suggest that the Nc, in response to the presentation of encoded objects, reflects recognition memory processes. We claim that the Nc is sensitive to familiarity or recognition and it relates to top-down processes. The lack of difference between frequent and infrequent familiar stimuli could mean that the Nc component is related to declarative memory. The present study and the novel paradigm we introduced, is an initial step toward the use of the Nc component as an electrophysiological signature that can be used to tease apart whether recognition memory in infants, an essential and distinctive component of human social development, is based upon specific or general memories.

\section{Acknowledgement}

I.K. received funding from KMOP-4.2.1/B-10-2011-0002.

E.P. is a researcher in the International Centre for Language and Communicative Development (LuCiD) at Lancaster University. The support of the Economic and Social Research Council (ES/L008955/1) is gratefully acknowledged.

\section{References}

Ackles, P.K., 2008. Stimulus novelty and cognitive-related ERP components of the infant brain. Percept. Mot. Skills 106 (1), 3-20. http://dx.doi.org/10.2466/pms.106.1.3-20.

Ackles, P.K., Cook, K.G., 1998. Stimulus probability and event-related potentials of the brain in 6-month-old human infants: a parametric study. Int. J. Psychophysiol. 29 (2), 115-143. http://dx.doi.org/10.1016/S0167-8760(98)00013-0.

Bauer, P.J., Wenner, J.A., Dropik, P.L., Wewerka, S.S., 2000. Parameters of remembering and forgetting in the transition from infancy to early childhood. Monogr. Soc. Res. Child Dev. 65 (4), 1-204. 〈http://doi.org/10.1016/j.imlet.2014.04.001〉.

Bauer, P.J., Wiebe, S.A., Carver, L.J., Waters, J.M., Nelson, C. a., 2003. Developments in long-term explicit memory late in the first year of life: behavioral and electrophysiological indices. Psychol. Sci. 14 (6), 629-635. http://dx.doi.org/10.1046/j. 0956-7976.2003.psci 1476.x.

Bauer, P.J., Wiebe, S.A., Carver, L.J., Lukowski, A.F., Haight, J.C., Waters, J.M., Nelson, C. a., 2006. Electrophysiological indexes of encoding and behavioral indexes of recall: examining relations and developmental change late in the first year of life. Dev. Neuropsychol. 29 (2), 293-320. http://dx.doi.org/10.1207/s15326942dn2902 2.

Bushnell, I.W.R., 2001. Mother's face recognition in newborn infants: learning and memory. Infant Child Dev. 10 (1-2), 67-74. http://dx.doi.org/10.1002/icd.248.

Carver, L.J., Bauer, P.J., 1999. When the event is more than the sum of its parts: 9-montholds' long-term ordered recall. Memory 7 (2), 147-174. http://dx.doi.org/10.1080/ 741944070.

Carver, L.J., Bauer, P.J., Nelson, C.A., 2000. Associations between infant brain activity and recall memory. Dev. Sci. 3 (2), 234-246. http://dx.doi.org/10.1111/1467-7687. 00116.

Casler, K., Kelemen, D., 2005. Young children's rapid learning about artifacts. Dev. Sci. 8 (6), 472-480. http://dx.doi.org/10.1111/j.1467-7687.2005.00438.x.

Courchesne, E., Ganz, L., Norcia, A.M., 1981. Event-related brain potentials to human faces in infants. Child Dev. 52 (3), 804-811. (Retrieved from). 〈http://www.ncbi. nlm.nih.gov/pubmed/7285651>.

de Haan, M., Nelson, C., 1997. Recognition of the mother's face by six-month-old infants: a neurobehavioral study. Child Dev. 68 (2), 187-210. http://dx.doi.org/10.1111/j. 1467-8624.1997.tb01935.x.

de Haan, M., Nelson, C., 1999. Brain activity differentiates face and object processing in 6-month-old infants. Dev. Psychol. 35 (4), 1113-1121. http://dx.doi.org/10.1037/ 0012-1649.35.4.1113.

Fantz, R.L., 1964. Visual experience in infants: decreased attention to familiar patterns relative to novel ones. Science 146 (3644), 668-670. http://dx.doi.org/10.1126/ science.146.3644.668.

Gergely, G., Csibra, G., 2006. Sylvia's Recipe: the Role of Imitation and Pedagogy in the Transmission of Cultural Knowledge. Berg Publisher, Oxford.

Hunter, M.A., Ross, H.S., Ames, E.W., 1982. Preferences for familiar or novel toys: effect of familiarization time in 1-year-olds. Dev. Psychol. 18 (4), 519-529. http://dx.doi. org $/ 10.1037 / 0012-1649.18 .4 .519$.

Koski, J., Olson, I.R., Newcombe, N.S., 2013. Tracking the eyes to see what children remember. Memory 21 (3), 396-407. http://dx.doi.org/10.1080/09658211.2012. 735241.

Nelson, C.A., Collins, P.F., 1991. Event-related potential and looking-time analysis of infants' responses to familiar and novel events: implications for visual recognition memory. Dev. Psychol. 27 (1), 50-58. http://dx.doi.org/10.1037/0012-1649.27. 1.50 .

Nelson, C.A., Collins, P.F., 1992. Neural and behavioral correlates of visual recognition memory in 4- and 8-month-old infants. Brain Cogn. 19 (1), 105-121. http://dx.doi. org/10.1016/0278-2626(92)90039-O. 
Reynolds, G.D., Courage, M.L., Richards, J.E., 2010. Infant attention and visual preferences: converging evidence from behavior, event-related potentials, and cortica source localization. Dev. Psychol. 46 (4), 886-904. http://dx.doi.org/10.1037/ a0019670.

Reynolds, G.D., Richards, J.E., 2005. Familiarization, attention, and recognition memory in infancy: an event-related potential and cortical source localization study. Dev. Psychol. 41 (4), 598-615. http://dx.doi.org/10.1037/0012-1649.41.4.598.

Richards, J.E., 2003. Attention affects the recognition of briefly presented visual stimuli in infants: an ERP study. Dev. Sci. 6 (3), 312-328. http://dx.doi.org/10.1111/14677687.00287.

Richards, J.E., Reynolds, G.D., Courage, M.L., 2010. The Neural Bases of Infant Attention. Current Directions Psychol. Sci. 19 (1), 41-46. http://doi.org/10.1177/ 0963721409360003.

Richmond, J., Colombo, M., Hayne, H., 2007. Interpreting visual preferences in the visual paired-comparison task. J. Exp. Psychol.: Learn. Mem. Cogn. 33 (5), 823-831. http:// dx.doi.org/10.1037/0278-7393.33.5.823.

Richmond, J., Nelson, C.A., 2009. Relational memory during infancy: evidence from eye tracking. Dev. Sci. 12 (4), 549-556. http://dx.doi.org/10.1111/j.1467-7687.2009. 00795.x.

Roder, B.J., Bushneil, E.W., Sasseville, A.M., 2000. Infants' preferences for familiarity and novelty during the course of visual processing. Infancy 1 (4), 491-507. http://dx.doi. org/10.1207/S15327078IN0104_9.

Tomasello, M., 1999. The cultural ecology of young children's interactions with objects and artifacts. In: Winograd, E., Fivush, R., Hirst, W. (Eds.), Ecological Approaches to Cognition: Essays in honor of Ulric Neisser. Erlbaum, Mahwah, NJ, pp. 153-170.

Wiebe, S.A., Cheatham, C.L., Lukowski, A.F., Haight, J.C., Haight, A.J., Bauer, P.J., 2006. Infants' ERP responses to novel and familiar stimuli change over time:time: implications for novelty detection and memory. Infancy 9 (1), 21-44. http://dx.doi.org/ 10.1207/s15327078in0901_2. 